\title{
Optimization of Effective Atom Centered Potentials for London Dispersion Forces in Density Functional Theory
}

\author{
O. Anatole von Lilienfeld, Ivano Tavernelli, and Ursula Rothlisberger* \\ Ecole Polytechnique Fédérale de Lausanne, Institut des Sciences et Ingénierie Chimiques, \\ EPFL-BCH, CH-1015 Lausanne, Switzerland \\ Daniel Sebastiani \\ Max-Planck-Institut für Polymerforschung, Ackermannweg 10, D-55128 Mainz, Germany
}

(Received 16 June 2004; published 7 October 2004)

\begin{abstract}
We add an effective atom-centered nonlocal term to the exchange-correlation potential in order to cure the lack of London dispersion forces in standard density functional theory. Calibration of this longrange correction is performed using density functional perturbation theory and an arbitrary reference. Without any prior assignment of types and structures of molecular fragments, our corrected generalized gradient approximation density functional theory calculations yield correct equilibrium geometries and dissociation energies of argon-argon, benzene-benzene, graphite-graphite, and argon-benzene complexes.
\end{abstract}

DOI: 10.1103/PhysRevLett.93.153004

PACS numbers: $31.15 . \mathrm{Md}, 71.15 . \mathrm{Mb}$

London dispersion forces are crucial for many fundamental molecular processes such as the interactions between rare gas atoms, the formation of tertiary and quaternary structures of biomolecules, the packing of molecular crystals, and the intercalation of drugs into DNA, to name only a few. These forces belong to the group of weak long-range van der Waals (vdW) forces: they represent the attractive interaction between selfinduced instantaneous dipole moments of ground state electron distributions [1]. Despite their obvious importance, it is not yet generally possible to correctly describe these nonlocal correlation effects within density functional theory (DFT) calculations with purely local exchange-correlation functionals [2-5]. Introduction of nonlocality into a "vdW functional" as proposed in Refs. [6,7] or by electron density partitioning [8] can abolish this deficiency but usually implies the artificial assignment of molecular fragments. Alternatively, Kohn et al. [5] or Misquitta et al. [9] proposed a general scheme for the calculation of dispersion forces with DFT but only at prohibitive computational costs. As a consequence, empirical atom-atom based correction terms of the $C_{6} / R^{6}$-type are frequently used instead $[4,10]$. Though very successful in many cases [11], preliminary calculations and validations are necessary to determine the values of these $C_{6}$ coefficients. In addition, for each pair of fragments or atom types individual damping functions have to be identified to account for the correct repulsive short-range behavior, rendering this approach somewhat cumbersome. Furthermore, empirical atom-atom potentials solely act on the ionic cores and do not influence the electronic structure; i.e., they do not correct any electronic properties.

Here, we propose to construct an effective potential consisting of optimized nonlocal higher angular momen- tum dependent terms for all atoms in the system in order to compensate for the absence of dispersion forces in a generalized gradient approximation (GGA) functional. Thus, instead of approximating the attractive long-range electron density correlation by an atom-atom interaction, we model vdW forces by an atom-electron interaction, mediated by appropriate nonlocal effective core potential (ECP) projectors which are obtained from our optimization scheme. We group this additional contribution together with the GGA exchange-correlation potential to form an extended exchange-correlation potential, $\hat{v}_{\mathrm{xc}}^{\text {ext }}=$ $\hat{v}_{\mathrm{xc}}^{\mathrm{GGA}}+\hat{v}^{\mathrm{eff}}$. For the calibration of the atom-projected effective potentials, we use accurate large basis set second order Møller-Plesset perturbation theory (MP2) results for typical weakly bonded systems as a reference. To this purpose, we have chosen molecular complexes which are well known to be bonded solely due to dispersion forces. We use the GGA functional of Becke, Lee, Yang, and Parr (BLYP) [12,13], and MP2 as a reference. This is an arbitrary choice; our approach could equally well be applied in the context of any other exchange-correlation functional and any other arbitrarily exact reference. In this study, we consider references at the full MP2 level of theory to be a reasonable compromise between accuracy and computational cost. High accuracy methods like coupled cluster theory or self-consistent field response methods (e.g., coupled perturbed Hartree-Fock) are desirable as benchmarks, but unfortunately, for larger systems, theoretical treatments beyond MP2 rapidly become computationally intractable.

In electronic structure theory atomic ECPs represent the potential of nuclei and their core electrons $[14,15]$. In particular, DFT calculations using plane wave basis sets are unfeasible without this technique. Initially introduced in an empirical form in 1970 [16] and later in a 
first-principles formulation [17], ECPs have been subject to continuous development ever since [18]. Recently, Goedecker et al. [19] published a library of analytic, separable, norm conserving ab initio pseudopotentials, which contain local and nonlocal, angular momentum $l$ dependent, terms of the form $V^{\mathrm{ECP}}\left(\mathbf{r}, \mathbf{r}^{\prime}\right)=V^{(\mathrm{loc})}(\mathbf{r}) \times$ $\delta\left(\mathbf{r}-\mathbf{r}^{\prime}\right)+\sum_{l} V_{l}^{(\mathrm{nl})}\left(\mathbf{r}, \mathbf{r}^{\prime}\right)$. Their local part consists of an error function and a Gaussian while the nonlocal terms consist of Gaussian-type radial projectors $p$ for each angular momentum channel $l$ :

$$
V_{l}^{(\mathrm{nl})}\left(\mathbf{r}, \mathbf{r}^{\prime}\right)=\sum_{m=-l}^{+l} Y_{l m}(\hat{\mathbf{r}}) \sum_{j, h=1}^{3} p_{l h}(r) h_{l h j} p_{l j}\left(r^{\prime}\right) Y_{l m}^{*}\left(\hat{\mathbf{r}}^{\prime}\right),
$$

where $p_{l h}(r) \propto r^{l+2(h-1)} \exp \left[-r^{2} /\left(2 r_{l}^{2}\right)\right], r=\left|\mathbf{r}-\mathbf{R}_{\mathrm{I}}\right|$ on the position $\mathbf{R}$ of nucleus I, $\hat{\mathbf{r}}$ is the unit vector in the direction of $\mathbf{r}$, and $Y_{l m}$ denotes a spherical harmonic. The parameters $\left\{\ldots, h_{l h j}, r_{l}, \ldots\right\}$ of these pseudopotentials (called $\left\{\sigma_{i}\right\}$ in the following) are generated by iteratively minimizing a penalty functional which expresses the deviations of the Kohn-Sham (KS) pseudo-orbitals from their all-electron counterparts.

The spirit of this generation procedure has motivated us to design effective atom-centered potentials by considering not only atomic but also complex molecular electronic properties as target quantities in the penalty functional. In our approach an analogous iterative minimization of a penalty functional $(\mathcal{P})$ is performed, where $\mathcal{P}$ is designed in such a way that it penalizes deviations from molecular properties (e.g., the electronic density) with respect to experimental or theoretical references.

The penalty functional $\mathcal{P}\left(\left\{\sigma_{i}\right\}\right)$ may depend on all quantities that can be expressed in terms of the KS orbitals. It can thus describe any arbitrary molecular property such as electronic densities $n(\mathbf{r})$ and multipole moments, as well as ionic forces $\mathbf{F}_{\text {Ions }}$ or energies. Therefore, $\mathcal{P}$ depends only indirectly (i.e., via the KS orbitals) on the ECP parameters $\left\{\sigma_{i}\right\}$.

In this way an additional effective potential can be introduced that has formally the same form as the ionelectron interaction potential, i.e., a linear combination of atom-centered nonlocal potentials. It can thus be treated with the usual computational machinery at negligible additional cost. However, the number and magnitude of all the parameters of this effective atom based nonlocal potential are freely tunable; the potential, e.g., can be chosen to act on a different characteristic length scale than the localized electron-core interaction. Our approach is therefore closely related to the optimized effective potential (OEP) method in DFT [20-24] where an orbital dependent external effective potential is optimized. In the case that $\mathcal{P}$ is designed to minimize the total energy of the system the scheme introduced here can be reduced to OEP.
We illustrate our procedure using a functional form dependent only on the electronic density $n(\mathbf{r})=$ $\sum_{k=1}^{N}\left|\phi_{k}(\mathbf{r})\right|^{2}, N$ being the number of the occupied KS orbitals $\phi_{k}$.

$$
\mathcal{P}\left[n\left(\mathbf{r},\left\{\sigma_{i}\right\}\right)\right]=\int d^{3} r w(\mathbf{r}) \mathcal{F}(n(\mathbf{r})),
$$

with a weighting function $w$ and a penalty function $\mathcal{F}$. Equation (2) is minimized by following the gradient of $\mathcal{P}$ with respect to the parameters $\sigma_{j}$ :

$$
\frac{d \mathcal{P}}{d \sigma_{j}}=\int d^{3} r w(\mathbf{r}) \frac{\partial \mathcal{F}}{\partial n(\mathbf{r})} \frac{d n(\mathbf{r})}{d \sigma_{j}} .
$$

The derivatives $d n(\mathbf{r}) / d \sigma_{j}$, which we denote $n_{j}^{(1)}(\mathbf{r})$, represent the linear response of the density induced by the parameter variation $\sigma_{j} \mapsto \sigma_{j}+d \sigma_{j}$. This density response can be computed through linear response theory, where the perturbation Hamiltonian is given by the change in the effective potential due to the variation in $\sigma_{j}: \hat{\mathcal{H}}_{j}^{\prime}=\partial \hat{V}^{\mathrm{ECP}}\left(\left\{\sigma_{i}\right\}\right) / \partial \sigma_{j}$. The density functional perturbation theory module [25] of the program CPMD [26] can be used to determine the vector of response densities $\left\{n_{j}^{(1)}(\mathbf{r})\right\}$. For all the DFT calculations presented in this Letter, we used CPMD [26], a plane wave cutoff of $100 \mathrm{Ry}$. The penalty function $\mathcal{F}$ and an appropriate weighting function $w$ [Eq. (2)] have to be chosen specifically for each application. To obtain an attractive dispersion interaction, we choose the penalty functional in Eq. (2) to be a sum of energy dependent and ionic force dependent terms (both functionals of the electron density):

$$
\mathcal{P}^{\mathrm{disp}}\left(\mathbf{R}^{\mathrm{ref}}\right)=\left|E^{\mathrm{ref}}\left(\mathbf{R}^{\mathrm{ref}}\right)-E\left(\mathbf{R}^{\mathrm{ref}}\right)\right|^{2}+\sum_{\mathrm{I}}^{N_{\mathrm{ions}}} w_{\mathrm{I}}\left|\mathbf{F}_{\mathrm{I}}\left(\mathbf{R}^{\mathrm{ref}}\right)\right|^{2},
$$

where $w(\mathbf{r})$ of Eq. (2) becomes $\sum_{\mathrm{I}} w_{\mathrm{I}} \delta\left(\mathbf{r}-\mathbf{R}_{\mathrm{I}}\right), w_{\mathrm{I}}=0$ or $w_{\mathrm{I}}=1$ select specific atoms for the optimization process, and $\mathbf{F}_{\mathrm{I}}$ and $E$ are the ionic forces and the energy of interaction at the reference geometry $\mathbf{R}^{\text {ref }}$, respectively. $E^{\text {ref }}$ corresponds to the reference dispersion interaction energy. By tuning the height $\left(\sigma_{1}=h_{l 11}\right)$ and the width $\left(\sigma_{2}\right)$ of an additional projector $\left[p_{l 1}(r) \propto r^{2} \exp \left(-r^{2} /\right.\right.$ $\left.\left(2 \sigma_{2}^{2}\right)\right)$ ], we minimize $\mathcal{P}^{\text {disp }}$ in such a way that the reference location and depth of the interaction energy minimum are reproduced for the calibration system. The choice to augment the ECPs by calibrating only one additional polarization channel is not crucial and could easily be extended to other angular momentum channels.

As a test case, we have calibrated dispersion-optimized effective potentials for carbon and argon. In the case of (aromatic) carbon, we use as a reference system the benzene dimer in its parallel sandwich configuration, as presented in the left graph of Fig. 1, while for argon we use the argon dimer (shown in the left graph of Fig. 2). For 


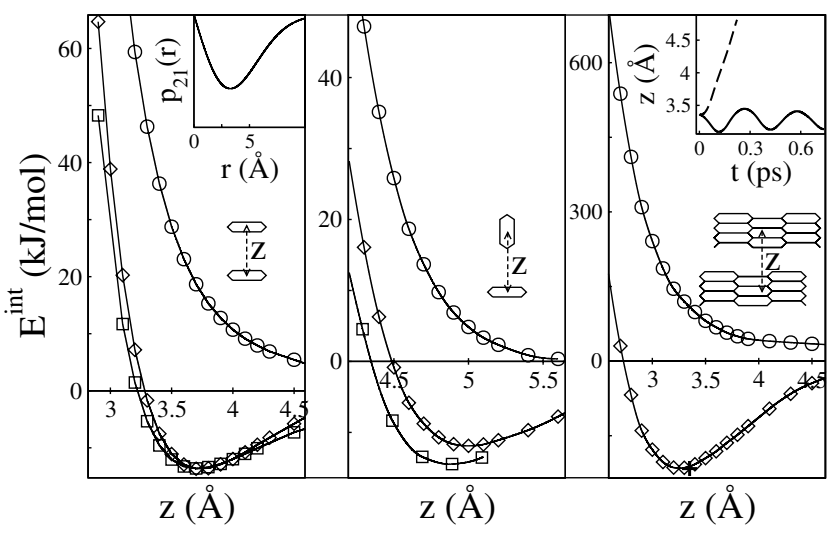

FIG. 1. In the inset of the left graph the additional $d$-channel projector $p_{21}(r)$ [Eq. (1)] is depicted in arbitrary units as a function of $r$, the minimum is $\approx 3.3 \AA$. Potential energy curves of the total energy of interaction $\left(E^{\text {int }}=E^{\text {Dimer }}-2 E^{\text {Monomer }}\right)$ are plotted as a function of the distance $z$. $\bigcirc$ corresponds to normal ECPs, $\diamond$ to the optimized ECP (OECP), and $\square$ to MP2 data from [29]. The experimental value for the interlayer distance and energy of interaction of two graphite sheets is marked by a cross. The left hand panel shows the curve for calibration. In atomic units the calibrated values of the additional $d$ channel for carbon are $\sigma_{1}=-0.00352$ and $\sigma_{2}=$ 3.280. The other two graphs represent results for the T-shaped configuration of benzene (middle panel) and for the slab of graphite (right hand panel) using the same values $\sigma_{1}, \sigma_{2}$. In the inset of the right hand panel the averaged interlayer distance $z$ is presented during first-principles BOMD using the normal ECP (dotted line) and the OECPs (continuous line).

benzene $w_{\mathrm{I}}$ was chosen to be zero in the case of hydrogen, and one in the case of carbon, while for $\operatorname{argon} w_{\mathrm{I}}=1$.

The resulting values for $\sigma_{1}$ and $\sigma_{2}$ create a weak longrange attractive potential. In the inset of the left graph of Fig. 1 the applied additional projector for carbon $p_{21}(r)$ is plotted against $r$. It is interesting to note that the minimum of this attractive potential lies at $3.3 \AA$ which is close to the equilibrium separation. However, the magnitude $\sigma_{1}$ remained so small that, e.g., the geometry of an isolated benzene monomer is not distorted (the average changes of the bond lengths are only $\approx 0.01 \AA$ ). Moreover, electronic quantities of the monomers remain basically unchanged, e.g., the static polarizability ( $\alpha$ ) of argon or the quadrupole moment $Q$ and the static polarizability anisotropy ( $\left.\Delta \alpha=\alpha_{y y}-\alpha_{z z}\right)$ of benzene (Table I).

Using the upgraded carbon ECPs, we computed interaction energies for a T-shaped benzene dimer configuration and for a slab made of two layers of graphene. The results are presented in Fig. 1 and show an astonishing transferability of the calibrated ECPs. For the T-shaped benzene dimer the results compare very well to the corresponding MP2 calculations from [29] which use the same basis set as those we have used for the calibration in the sandwich configuration. To compute graphite, we arranged 64 atoms in a slab made of two layers, and
TABLE I. Calculated polarizabilities, quadrupole and dipole moments for BLYP calculations with the unchanged ECPs, the optimized ECPs (OECP), and MP2 calculations for comparison. $b z$ represents benzene, and bz-Ar the benzene-argon complex. All values are in atomic units.

\begin{tabular}{lccccc}
\hline \hline Method & $\alpha_{\mathrm{Ar}}$ & $Q_{\mathrm{bz}}{ }^{\mathrm{a}}$ & $\Delta \alpha_{\mathrm{bz}}{ }^{\mathrm{a}}$ & $\alpha_{\mathrm{bz}-\mathrm{Ar}}{ }^{\mathrm{a}}$ & $\mu_{\mathrm{bz}-\mathrm{Ar}}{ }^{\mathrm{a}}$ \\
\hline ECP & 12.30 & -5.35 & 39.18 & 55.0 & 0.047 \\
OECP & 12.31 & -5.50 & 38.45 & 58.1 & 0.035 \\
MP2 & $11.15^{\mathrm{b}}$ & $-6.46^{\mathrm{b}}$ & $35.07^{\mathrm{c}}$ & $59.2^{\mathrm{b}}$ & $0.037^{\mathrm{b}}$ \\
\hline
\end{tabular}

${ }^{\mathrm{a}} Q=\left\langle z^{2}-\frac{1}{2} x^{2}-\frac{1}{2} y^{2}\right\rangle, \quad \Delta \alpha_{\mathrm{bz}}=\alpha_{z z}^{\mathrm{bz}}-\alpha_{y y}^{\mathrm{bz}}, \quad \alpha_{\mathrm{bz}-\mathrm{Ar}}=\alpha_{z z}^{\mathrm{bz}-\mathrm{Ar}}$. ${ }^{\mathrm{b}} \mathrm{MP} 2$ results from Ref. [27].

${ }^{\mathrm{c}} \mathrm{MP} 2$ results from Ref. [28].

applied periodic boundary conditions within the $x y$ plane of the graphite sheets. The system was isolated in the $z$ direction which is normal to the planes. For graphene layers no MP2 data are available for comparison. However, the calculated equilibrium distance of $3.3 \AA$ and interaction energy of $32 \mathrm{meV} /$ atom are in surprising agreement with the experimental interlayer distance and the experimental energy of interaction between two graphene sheets, $3.35 \AA$ and $35 \pm 10 \mathrm{meV} /$ atom [30], respectively. Using the same setup as for the graphene single point calculations, we present results for $0.7 \mathrm{ps} N V T$ Born-Oppenheimer molecular dynamics (BOMD) at $300 \mathrm{~K}$ in Fig. 2. They confirm our findings that using the upgraded ECPs the average distance between the sheets is $\approx 3.3 \AA$ while using normal ECPs the sheets simply dissociate, as expected from the purely repulsive interaction energy curve.

In order to extend the assessment of the transferability to combinations of different atoms, we have investigated the benzene-argon dimer. Without any further tuning of the upgraded ECP parameters, we have computed the potential energy curve for the benzene-argon dimer in its $C_{6 v}$ symmetry. The resulting curve is presented in Fig. 2 and reproduces the equilibrium distance and interaction energy of corresponding MP2 calculations [27]. Also electronic properties such as the polarizability $\left(\alpha_{\mathrm{bz}-\mathrm{Ar}}\right)$ due to an electric field parallel to the $C_{6 v}$ symmetry axis and the small permanent dipole moment $\left(\mu_{\mathrm{bz}-\mathrm{Ar}}^{z}\right)$ induced by the mutual polarization of the monomers show good agreement with MP2 predictions (Table I). The inset of the right graph of Fig. 2 depicts the difference of the electron density when upgraded ECPs are used instead of conventional ECPs. The optimized ECPs decrease electron density in the short-range regions of the atoms and increase it in the outer core regions, thus generating a small additional electron density overlap and thereby weak bonding.

All our results suggest that it is possible to correct for the lack of electron correlation across low density regions with dispersion-optimized atom based effective potentials. We think that this scheme has advantages with 

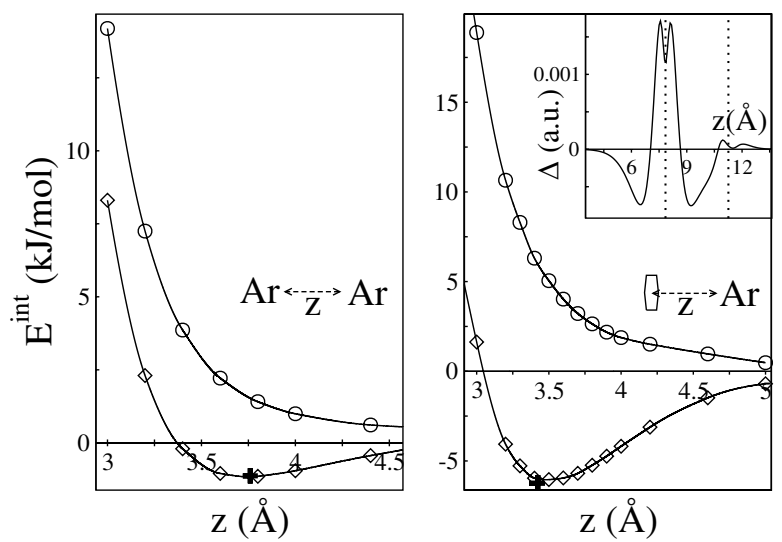

FIG. 2. For the argon-argon and the benzene-argon dimer potential energy curves of the total energy of interaction ( $\left.E^{\text {int }}=E^{\text {Dimer }}-2 E^{\text {Monomers }}\right)$ are plotted as a function of the distance $z$. $\bigcirc$ corresponds to the normal ECPs, $\diamond$ to the optimized ECP (OECP), and the MP2 results [27] for the interlayer distances and energies of interaction are marked by a cross. In atomic units the calibrated values of the additional $f$ channel for argon are $\sigma_{1}=-0.00206$ and $\sigma_{2}=2.902$. In the inset of the right hand panel the $C_{6 v}$ symmetry axis $z$ is plotted versus $\Delta=\int d x d y\left[n^{\text {norm }}(\mathbf{r})-n^{\text {opt }}(\mathbf{r})\right]$ (the differences between integrated $x y$ planes of the electron density of the benzeneargon dimer at equilibrium distance computed with the normal ECPs and the OECPs, respectively). The dotted lines show the position of the moieties: benzene (the molecular plane is perpendicular to $z$ ) is at $7.85 \AA$ on the $z$ axis, and argon is at $11.26 \AA$.

respect to the usual empirical corrections for two reasons. First, the improved electronic properties (dipole moment, quadrupole moment, and polarizability) indicate that due to the nonlocality of the ECP projectors, the valence wave functions reproduce more of the characteristics of dispersion interactions than a simple additive atom-atom based correction. Second, properly calibrated and transferable atomic dispersion calibrated ECPs no longer need any artificial a priori assignment of interacting groups or atoms. Therefore, we have shown a possibility to generally include dispersion forces in all DFT first-principles calculations at essentially no additional cost. We conclude that our scheme to optimize effective external potentials can be used to improve the description of molecular properties within DFT. We have illustrated the potential of our method by means of successfully modeling attractive long-range vdW forces within ECP based GGA DFT calculations.

The authors thank A. Laio, P. Cummins, M. Sprik, T. Wesolowski, G. von Freymann and M. Parrinello for discussions. A. v. L is indebted to M. O. Sinnokrot and C. D. Sherrill for comments and additional MP2 reference data points of the benzene dimer and acknowledges support from the Swiss National Science Foundation, Grant No. 510573.
*Electronic addresses: ursula.roethlisberger@epfl.ch http://lcbcpc21.epfl.ch

[1] D. P. Craig and T. Thirunamachandran, in Molecular Quantum Electrodynamics (Dover Publications, Inc., Mineola, NY, 1998).

[2] S. Kristyán and P. Pulay, Chem. Phys. Lett. 229, 175 (1994).

[3] J. M. Pérez-Jordá and A. D. Becke, Chem. Phys. Lett. 233, 134 (1995).

[4] E J. Meijer and M. Sprik, J. Chem. Phys. 105, 8684 (1996).

[5] W. Kohn, Y. Meir, and D. E. Makarov, Phys. Rev. Lett. 80, 4153 (1998).

[6] H. Rydberg, B. I. Lundqvist, D. C. Langreth, and M. Dion, Phys. Rev. B 62, 6997 (2000).

[7] H. Rydberg, M. Dion, N. Jacobsen, E. Schröder, P. Hyldgaard, S. I. Simak, D. C. Langreth, and B. I. Lundqvist, Phys. Rev. Lett. 91, 126402 (2003).

[8] T. A. Wesolowski and F. Tran, J. Chem. Phys. 118, 2072 (2003).

[9] A. J. Misquitta, B. Jeziorski, and K. Szalewicz, Phys. Rev. Lett. 91, 033201 (2003).

[10] R. LeSar, J. Phys. Chem. 88, 4272 (1984).

[11] X. Wu, M. C. Vargas, S. Nayak, V. Lotrich, and G. Scoles, J. Chem. Phys. 115, 8748 (2001).

[12] A. D. Becke, J. Chem. Phys. 88, 2547 (1988).

[13] C. Lee, W. Yang, and R. G. Parr, Phys. Rev. B 37, 785 (1988).

[14] H. Hellmann, J. Chem. Phys. 3, 61 (1935).

[15] U. von Barth and C. D. Gelatt, Phys. Rev. B 21, 2222 (1980).

[16] V. Heine and D. Weaire, Solid State Phys. 24, 249 (1970).

[17] G. B. Bachelet, D. R. Hamann, and M. Schluter, Phys. Rev. B 26, 4199 (1982).

[18] W. E. Pickett, Comput. Phys. Rep. 9, 115 (1989).

[19] C. Hartwigsen, S. Goedecker, and J. Hutter, Phys. Rev. B 58, 3641 (1998).

[20] R. T. Sharp and G. K. Horton, Phys. Rev. 90, 317 (1953).

[21] A. Görling and M. Levy, Phys. Rev. A 50, 196 (1994).

[22] L. Fritsche and J. M. Yuan, Phys. Rev. A 57, 3425 (1998).

[23] R. Colle and R. K. Nesbet, J. Phys. B 34, 2475 (2001).

[24] W. Yang and Q. Wu, Phys. Rev. Lett. 89, 143002 (2002).

[25] A. Putrino, D. Sebastiani, and M. Parrinello, J. Chem. Phys. 113, 7102 (2000).

[26] J. Hutter et al., Computer code CPMD, version 3.8, Copyright IBM Corp., and MPI-FKF Stuttgart 1990-2003, http://www.cpmd.org.

[27] W. Klopper, H. P. Lüthi, T. Brupbacher, and A. Bauder, J. Chem. Phys. 101, 9747 (1994).

[28] H. Soscún, J. Hernández, R. Escobar, C. Toro-Mendoza, Y. Alvarado, and A. Hinchliffe, Int. J. Quantum Chem. 90, 497 (2002).

[29] M. O. Sinnokrot, E. F. Valeev, and C. D. Sherrill, J. Am. Chem. Soc. 124, 10887 (2002).

[30] L. X. Benedict, N. G. Chopra, M. L. Cohen, A. Zettl, S. G. Louie, and V. H. Crespi, Chem. Phys. Lett. 286, 490 (1998). 